\title{
Ventilation/perfusion tomography - V/P-SPECT vs planar technique
}

\author{
Amela Begic ${ }^{1}$, Emina Opanković ${ }^{1}$, Sadžida Begović-Hadžimuratović ${ }^{1}$, Miran Konjić ${ }^{2}$, Senahid \\ Krekić $^{2}$ \\ 1. Clinic for Nuclear Medicine, University Hospital of Sarajevo, Sarajevo, Bosnia and Herzegovina. 2. Clinic for Pulmology, \\ University Hospital of Sarajevo, Sarajevo, Bosnia and Herzegovina.
}

Correspondence: Amela Begic. Address: Clinic for Nuclear Medicine, University Hospital of Sarajevo, Sarajevo, Bosnia and Herzegovina. Email: amelab709@gmail.com

Received: J une 4, 2014

DOI: $10.5430 /$ crim.v1n2p205
Accepted: June 8, 2014

Online Published: July 8, 2014

\begin{abstract}
Pulmonary embolism (PE) can only be diagnosed with imaging techniques, which in practice is performed using ventilation/perfusion scintigraphy(V/P scintigraphy) or multi-detector computed tomography of the pulmonary arteries (MDCT). The basic principle for the diagnosis of $\mathrm{PE}$ based upon $\mathrm{V} / \mathrm{P}$ scintigraphy is to recognize lung segments or subsegments without perfusion but preserved ventilation, i. e. mismatch. Ventilation/ perfusion single photon emission tomography - V/P SPECT has during the last 10 years started to replace V/P planar based on higher sensitivity, specificity and low non-diagnostic findings.

A twenty seven year old woman was suspected for PE when presented to the out-patient clinic due to dyspnea, frequent yawing, oppression in the throat, 3 days after being treated of superficial varices cruris with foam sclerotherapy - UGFS. She was first examined with chest x-ray, MDCT, hearth ultrasonography and V/P planar. V/P planar was reported as very low probability for PE. All the other initial examinations were normal. Patient symptoms did not decline, and reffering clinitian put her on therapy with LMWH. Fifth days later reffering doctor recomended V/P SPECT. V/P SPECT has shown billateral PE, with the cca 35\% extent of PE. Patient was followed up clinically and with V/P SPECT one month later. The control V/P SPECT was normal. This case presented importance of high clinical probability and value of V/P SPECT over another imaging technique for detection of PE.
\end{abstract}

\section{Keywords}

Pulmonary embolism, Ventilation/perfusion single photon tomography, V/P-SPECT, Planar ventilation/perfusion scintigraphy-V/P planar, Follow up

\section{Introduction}

Pulmonary embolism (PE) can only be diagnosed with imaging techniques, which in practice is performed using ventilation/perfusion scintigraphy (V/P scintigraphy), or multi-detector computed tomography of the pulmonary arteries $(\mathrm{MDCT}){ }^{[1]}$. The basic principle for diagnosis of PE is based upon V/P scintigraphy to recognize lung segments or sub-segments without perfusion but preserved ventilation, i.e. mismatch ${ }^{[1]}$. PE should be report when mismatch of more than one sub-segment is found ${ }^{[1]}$. V/P scintigraphy for diagnosis of $\mathrm{PE}$ is universally available but imaging protocols and 
interpretative strategies show large variation ${ }^{[1]}$. Planar V/P scintigraphy was until 1990s the method of choice for studying patients with suspected PE ${ }^{[2]}$. However, the large Prospective Investigation of Pulmonary Embolism Diagnosis (PIOPED I) showed a high number of non-diagnostic examinations (65\%) and the probabilistic interpretation criteria were confusing to the clinicians ${ }^{[3,4]}$. The advantage of three dimensional tomography over planar imaging for PE detection had already been shown in 1983 in study on dogs ${ }^{[5]}$. Later on, Bajc et al. validated V/P SPECT for diagnosis of PE using porcine model, and confirmed the superior value of V/P SPECT over V/Pplanar imaging ${ }^{[6]}$. Palmer et al. developed an efficient method for ventilation/perfusion single photon emission tomography (V/P SPECT) for clinical practice ${ }^{[7]}$. Moreover, the authors developed an algorithm to calculate the quotient between ventilation and perfusion and to present it as $\mathrm{V} / \mathrm{P}$ quotient images ${ }^{[7]}$. EANM guidelines recommend V/P-SPECT as a first choice method, due to the high negative predictive value, high sensitivity and specificity, feasibility in almost all patients and low radiation exposure ${ }^{[8-11]}$. Furthermore, the strengths of V/P SPECT is in the new interpretation criteria with clear answer to the clinicians regarding $\mathrm{PE}$, yes or no ${ }^{[1,12]}$. V/P SPECT reports overcome obsolete probabilistic criteria interpreted in PIOPED study ${ }^{[1]}$.

\section{Case presentation}

A twenty-seven year old woman was presented to the out-patient clinic three days after treatment of superficial varices cruris with foam sclerotherapy - UGFS. She was complaining of dyspnea, frequent yawing, oppression in the throat, but no chest pain. D-dimer were elevated (> 15000). Suspicion of pulmonary embolism (PE) was raised.

Patient was examined with chest x-ray, MDCT, and hearth ultrasonography, that all were normal. Despite normal findings patient complained on the same symptoms. This was the reason why responsible physician referred patient for V/P scintigraphy at our clinic. V/P planar was initially performed and was reported as very low probability for PE (see Figure 1a, 1b). Clinician has however, administrated small doses of LMWH (low molecular weight heparin) due to present clinical symptoms.

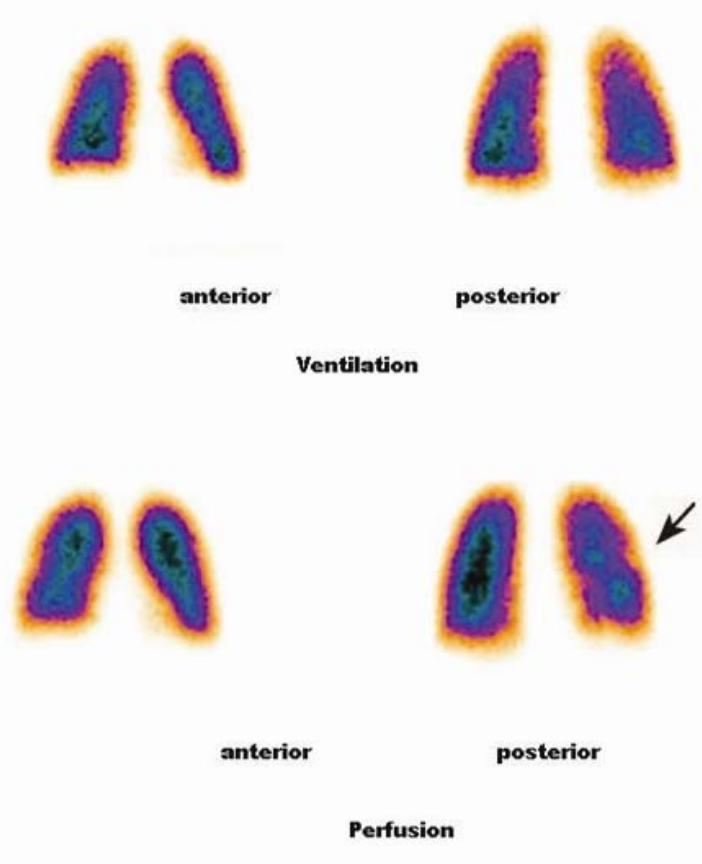

Figure 1a. V/P planar anterior and posterior projections: reduced perfusion in the right lower lobe with preserved ventilation.

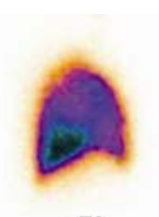

$\mathbf{R L}$

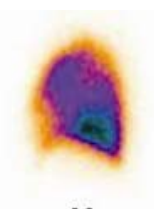

LL

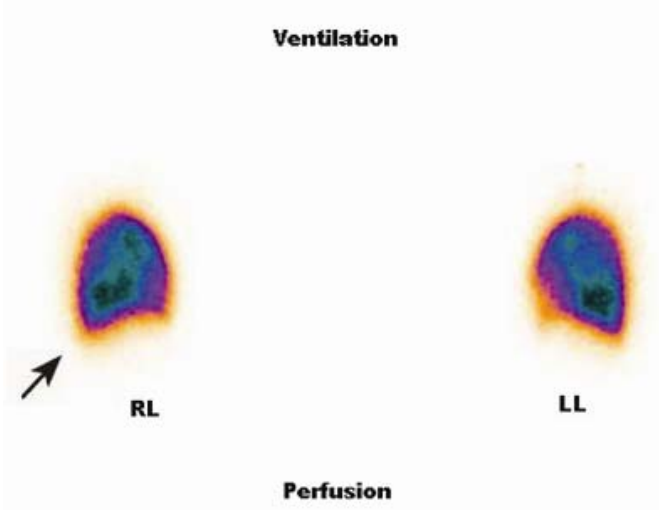

Figure 1b. V/P planar: Right lateral and left lateral projections: reduced perfusion in the right lower lobe with preserved ventilation. 
Due to the stagnant symptoms, the high clinical probability of PE was still present, why referring clinician recommended V/P SPECT fifth days after initial symptoms.

V/P SPECT showed bilateral PE, with the extent of PE ca 35\% (see Figure 2). Patient continued treatment with LMWH. Patient was followed-up clinically, and with V/P SPECT after one month. Eventually, the patient become free of symptoms and control V/P SPECT showed normalization of perfusion defects (see Figure 3). During further clinical controls patient was asymptomatic. Anticoagulant therapy was discontinued after 3 months.

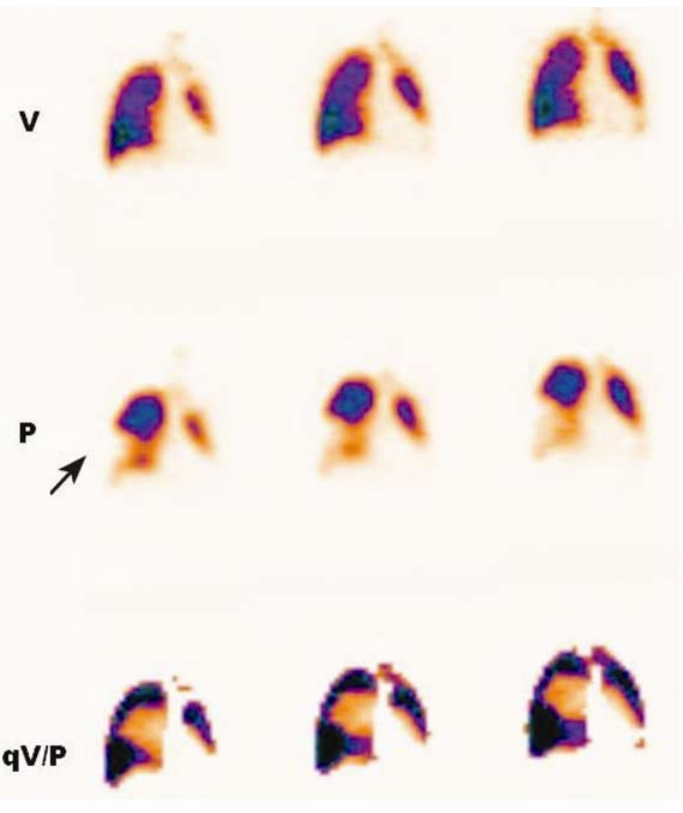

Figure 2. The frontal slices: absent and reduced perfusion in the right lower lobe (arrow). Ventilation is preserved in these areas.
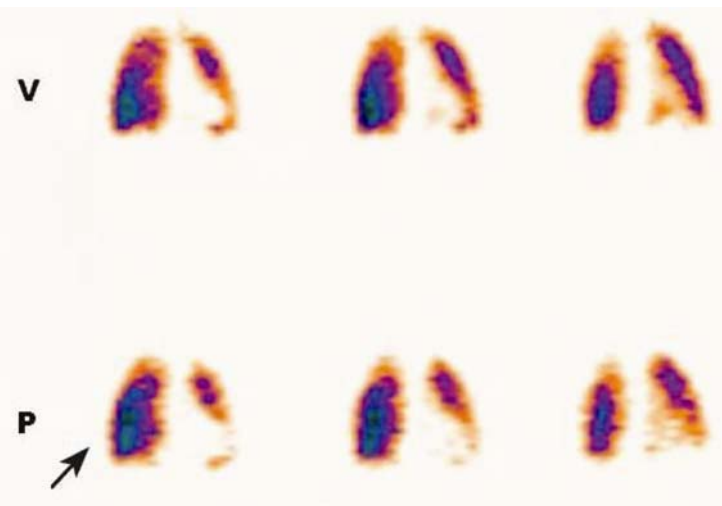

$\mathbf{q V} / \mathbf{P}$
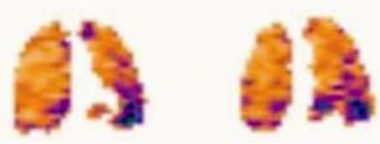

Figure 3. V/P SPECTshowed normalization of perfusion defectsone month later.

\section{Discussion}

Diagnosis of PE can only be confirmed and excluded with imaging techniques. MDCT is the most common recommended diagnostic procedure of first choice for the diagnosis of $\mathrm{PE}{ }^{[13]}$. The advantage of MDCT is the availability in nearly all medical centres. On the other hand the use of MDCT is contraindicated in patients with other diseases such as renal failure, critical illness, recent myocardial infarction or patients with allergy to the contrast medium ${ }^{[14,15]}$. The PIOPED II study has shown sensitivity of MDCT for PE of $83 \%$ excluding non-diagnostic studies. If non-diagnostic studies were included sensitivity decreased to $78 \%$. In the PIOPED II study the positive predictive value for a PE within lobar pulmonary artery was $97 \%$ but fell to $68 \%$ and $25 \%$ in segmental and sub-segmental pulmonary vessels, respectively ${ }^{[15]}$. In our case, the patient underwent MDCT as the first diagnostic procedure for diagnosis of PE, and was normal. As we mentioned, despite normal finding, clinical suspicion of PE persisted, and responsible physician needed further diagnostic evaluation.

Detection of ventilation and perfusion defects at the sub-segmental level is possible by planar imaging, but is considerably better by SPECT ${ }^{[1]}$. V/P SPECT images has documented value over planar images ${ }^{[6,16-20]}$ and over $\mathrm{CT}^{[8,10]}$.

In a pig model with artificial subsegmental emboli, the sensitivity of V/P planar was $67 \%$ and of V/P SPECT was $93 \%{ }^{[20]}$. In clinical studies, Bajc et al. revealed 53\% more mismatched regions with SPECT ${ }^{[22]}$. Reinartz et al. demonstrated also a 
higher number of perfusion defects by SPECT at the segmental and the sub-segmental level by $12.8 \%$ and $82.6 \%$ respectively ${ }^{[19]}$.

Comparing V/P planar and V/P SPECT Gutte et al. reported that V/P SPECT had a sensitivity of $100 \%$ and specificity of $87 \%$ while, V/P planar had a sensitivity of $64 \%$ and specificity of $72 \%{ }^{[17]}$. The value of V/P SPECT over planar images were recently confirmed by Quirice at al ${ }^{[23]}$. In a studied cohort 63 of $102 \mathrm{~V} / \mathrm{P}$ planar examinations $(62 \%)$ were non-diagnostic ${ }^{[23]}$. V/P SPECT allows better segmental definition of perfusion and ventilation defects and helped to significantly reduce the number of inconclusive results to $4.9 \%$. The sensitivity is improved by lessening the number of potential false negative results of the planar technique ${ }^{[23]}$. The high incidence of inconclusive studies for V/P planar is explained by the interpretation criteria applied ${ }^{[23]}$. Reinartz et al. found a sensitivity and specificity of 0.76 and 0.85 , respectively, with V/P planar compared to 0.97 and 0.91 with V/P SPECT ${ }^{[19]}$. In a study by Collart et al., V/P SPECT increased the specificity for PE from $78 \%$ to $96 \%$ at similar sensitivities ${ }^{[24]}$. Recent study by Gruning et. al. also confirmed high sensitivity of V/P SPECT of $95.7 \%$, specificity $98.6 \%$, positive predictive value $95.7 \%$. negative predictive value $98.6 \%{ }^{[13]}$. In our case V/P planar was performed before V/P SPECT because we were in transitional period of leaving planar scintigraphy and probabilistic interpretation criteria. Since this case V/P SPECT replaced fully planar technique and become primary diagnostic method for patient with suspected PE at our clinic. The findings are interpreted according to EANM guidelines ${ }^{[1,12]}$.

This case once again demonstrates the strength of V/P SPECT as conclusive imaging test, which provides clear visualization of pathological changes.In our opinion the patient had PE from the very beginning and was not diagnosed with the first choose methods. The similar results were described by Quirce et al. who showed advantage of V/P SPECT in three patients with normal planar technique,and concluded that the V/P SPECT technique makes an important diagnostic contribution by decreasing number of inconclusive reports ${ }^{[22]}$.

To achieve the clinical utility, interpretation of an imaging test should be confirmative or negative with respect to PE (PE: yes or no) and should not be based on probability categories ${ }^{[1,12]}$.

A case of the young women who developed PE showed clearly that it's a time to move from V/P planar and use V/P SPECT as a first choice method in patients with suspected PE. Furthermore, this case highlights the importance of clinical judgment and advantages of using principles of interpretation criteria recommended by EANM guidelines. Furthermore, V/P SPECT alows quantification of the PE extent which might be used to personalize treatment ${ }^{[6,15]}$.

\section{References}

[1] Bajc M, Neilly J.B, Miniati M, Schuemichen C, Meignan M, and Jonson B, "EANMquidelines for ventilation/perfusion scintigraphy: part 1. Pulmonary imaging with ventilation/perfusion single photon emission tomography", European Journal of Nuclear Medicine and Molecular Imaging. 2009; 36(8): 1356-1370. http://dx.doi.org/10.1007/s00259-009-1170-5

[2] Bajc M. and Jögi J. Quantitative Ventilation/Perfusion Tomography: the Foremost Technique for Pulmonary Embolism Diagnosis, Pulmonary Embolism, Dr. Ufuk Çobanoğlu (Ed.), ISBN: 978-953-51-0233-5, InTech. 2012. http://dx.doi.org/10.5772/32310 Available from:

http://www.intechopen.com/books/pulmonary-embolism/ventilation-perfusion-spect-first-hand-method-for-diagnosis-pulmonary -embolism

[3] Gray, H.W, McKillop, J.H, and Bessent, R.G. Lung scan reports: interpretation by clinicians. Nucl Med Commun. 1993; 14(11): 989-994. http://dx.doi.org/10.1097/00006231-199311000-00009

[4] The PIOPED investigators Value of the ventilation/perfusion scan in acute pulmonary embolism. Results of the prospective investigation of pulmonary embolism diagnosis (PIOPED). Jama. 1990; 263(20): 2753-2759. http://dx.doi.org/10.1001/jama.1990.03440200057023

[5] Osborne D.R, Jaszczak, R.J, Greer, K, Roggli, V, Lischko, M, et al. Detection of pulmonary emboli in dogs: comparation of single photon emission computed tomography, gamma camera imaging, and angiography. Radiology. 1983; 146(2): 493-497. 
[6] Bajc M, Bitzén U, Olsson B, Perez de Sá V, Palmer J, Jonson B. Lung ventilation/perfusion SPECT in the artificially embolized pig. J Nucl Med. 2002b May;43(5):640-7.

[7] PalmerJ, Bitzén U,Jonson B, and Bajc M. Comprehensive ventilation/perfusion SPECT. J Nucl Med. 2001; 42 (8): $1288-1294$.

[8] Bajc M, Olsson B, Palmer J, and Jonson B. Ventilation/perfusion SPECT for diagnostic of pulmonary embolism in clinical practice. Journal of internal medicine. 2008; 264(4): 379-387. http://dx.doi.org/10.1111/j.1365-2796.2008.01980.x

[9] Leblanc M, Leveillee F, and Turcotte E. prospective evaluation of the negative predictive value of V/Q SPECT using Technegas. Nucl Med Commun. 2007; 28(8): 667-672. http://dx.doi.org/10.1097/MNM.0b013e32827a8e99

[10] Gutte H, Mortensen J, Jensen C.V, Johnbeck C.B, von der Recke P, Petersen C.L, et al. Detection of Pulmonary Embolism with Combined Ventilation-Perfusion SPECT and Low-Dose CT: Head-to Head Comparation with Multidedector CT Angiography. J Nucl Med. 2009; 50: 1987-1992. http://dx.doi.org/10.2967/jnumed.108.061606

[11] Gruning T, Drake B.E, Farrell S.L, Nokes T. Three- year clinical experience with VQ SPECT for diagnosing pulmonary embolism: diagnostic performance. Clinical Imaging. Article in Press. http://dx.doi.org/10.1016/j.clinimag. 201404.003

[12] Schuemichen C. Pulmonary embolism: is multislice CT the method of choice? Against. Eur J Nucl Med Mol Imaging. 2005; 32: 107-12. http://dx.doi.org/10.1007/s00259-004-1679-6

[13] Bajc M, Neilly J.B, Miniati M, Schuemichen C, Meignan M, Jonson B. EANM guidelines for ventilation/perfusion scintigraphy. Part 2. Algorithms and clinical considerations for diagnosis of pulmonary emboli with V/P spect and MDCT. Eur J Nucl Med Mol Imaging. 2009; 36: 1528-1538. http://dx.doi.org/10.1007/s00259-009-1169-y

[14] Stein PD, Fowler SE, Goodman LR, Gottschalk A, Hales CA, Hull RD, et al. Multidetector computed tomography for acute pulmonary embolism. N Engl J Med. 2006; 354: 2317-27. http://dx.doi.org/10.1056/NEJMoa052367

[15] Sostman HD, Miniati M, Gottschalk A, Matta F, Stein PD, Pitstoseli M. Sensitivity and specificity of perfusion scintigraphy combined with chest radiography for acute pulmonary embolism in PIOPED II. J Nucl Med. 2008; 49: 1741-8. http://dx.doi.org/10.2967/jnumed.108.052217

[16] Bajc M, Olsson CG, Olsson B, Palmer J, Jonson B. Diagnostic evaluation of planar and tomographic ventilation/perfusion lung images in patients with suspected pulmonary emboli. Clin Physiol Funct Imaging. 2004 Sep; 24(5): 2. http://dx.doi.org/10.1111/j.1475-097X.2004.00546.x

[17] Gutte H, Mortensen J, Jensen CV, von der Recke P, Petersen CL, Kristoffersen US, et al. Comparasion of V/Q SPECT and planar V/Q lung scintigraphy in diagnosing acute pulmonary embolism. Nucl Med Commun. 2010 Jan; 31(1): 82-6. http://dx.doi.org/10.1097/MNM.0b013e3283336747

[18] Palla A, De Nitto P, Santolicandro A. Planar versus SPECT studies in lung disease. J Nucl Biol Med. 1994 Mar; $38(1)$ : $22-36$

[19] Reinartz P, Wildberger JE, Schaefer W, Nowak B, Mahnken AH, Buell U. Tomographic imaging in the diagnosis of pulmonari embolism: a comparation between V/Q lung scintigraphy in SPECT technique and multislice spiral CT. J Nucl Med. 2004 Sep; 45(9): 1501-8

[20] Begic A, Begic A, Kucukalic-Selimovic E, Zutic H, Skopljak-Beganovic A. Sreening of pulmonary embolism by ventilation/perfusion tomography-SPECT and comparation with planar scintigraphy: our experiences. Med Arh. 2008; 62: 220-222

[21] Bajc M. Value of ventilation/perfusion SPECT detecting extensive pulmonary embolism in a patients with pneumonia. Thromb Haemost. 2005; 93: 993-4.

[22] Bajc M, Olsson CG, Plamer J, Jonson B. Quantitative ventilation/perfusion SPECT (QV/P SPECT): a primary method for diagnosis of pulmonary embolism. In: Freeman LM,editor. Nuclear Medicine Annual. Philadelphia: Lippincott Williams \& Wilkins; 2004; 173-86.

[23] Quirice R, Ibanez-Bravo S, Jimenez-Bonilla J, Martinez-Rodriguez I, Martinez-Amador N, Ortega-Nava F, et al. Contribution of V/Q SPECT to planar scintigraphy in the diagnosis of pulmonary embolism. Rev Esp Med Nucl Imagen Mol. 2014; 33(3): 153-158

[24] Collart JO, Roelants V, Vanpee D, Lacrosse M, Trigaux JP, Delaunois L, et al. Is a lung perfusion scan obtained by using single photon emission computed tomography able to improve the radionuclide diagnosis of pulmonary embolism? Nucl Med Commun 2002; 23: 1107 -13. http://dx.doi.org/10.1097/00006231-200211000-00011 